\section{Oft keine Operation bei kleinen Nierentumoren}

Welches Vorgehen bei kleinen, asymptomatischen Nierentumoren das beste ist, wurde jetzt in einer Metaanalyse an 880 Patienten untersucht.

$\mathrm{K}$ leine, asymptomatische Nierentumoren werden häufig per Zufall bei der abdominellen Bildgebung entdeckt. Solche Geschwüre mit einer Größe von weniger als $4 \mathrm{~cm}$ bilden inzwischen etwa die Hälfte aller aufgespürten Nierentumoren, berichten Marc Smaldone und Mitarbeiter aus Philadelphia, PA/USA. In der Regel werden die Wucherungen entfernt, doch gerade bei älteren oder multimorbiden Patienten stellt sich die Frage, ob ihnen die Operation mehr schadet als der Tumor.

Um diese Frage zu klären, werteten die Onkologen um Smaldone in einer Metaanalyse Daten von 880 Patienten mit 936 Tumoren aus 18 Studien aus. In diesen wurde bei Patienten mit kleinen lokali- sierten Tumoren zunächst abgewartet, ob es zur Progression kommt, und erst dann operiert. Als Konsequenz wurden sie engmaschig radiologisch überwacht. Insgesamt metastasierten die Tumoren nur bei 18 Patienten (2\%), und dies im Schnitt erst 40 Monate nach der Diagnose.

Von sechs Studien mit 259 Patienten lagen auch individuelle Daten vor. Nach einer durchschnittlichen Beobachtungszeit von knapp drei Jahren zeigte ein Viertel der Tumoren überhaupt kein Wachstum. Insgesamt waren die Geschwüre ausgehend von etwas mehr als $2 \mathrm{~cm}$ bei der Diagnose nur um etwa 0,3 bis $0,4 \mathrm{~cm}$ pro Jahr gewachsen, was einer Volumenzunahme von etwas über $6 \mathrm{~cm}^{3}$ pro Jahr entsprach.
Bei keinem der Patienten in dieser Gruppe kam es zu Metastasen, allerdings wurden $45 \%$ der Tumoren letztlich doch operiert, zum größten Teil auf Wunsch der Patienten (58\%). Nur bei einem Drittel der Operierten war das rasche Wachstum der Tumoren der Grund für den Eingriff.

Fazit: Kontrolliertes Abwarten stellt eine gute Option für ältere oder schwer kranke Nierentumor-Patienten dar, bei denen es unwahrscheinlich ist, dass sie eine $\mathrm{Me}$ tastasierung des Tumors überhaupt erleben werden, oder bei denen aus anderen Gründen eine Operation problematisch erscheint. Die Tumorkontrolle per Bildgebung wird dann alle drei bis sechs Monate empfohlen

Thomas Müller

Smaldone MC et al. Small renal masses progressing to metastases under active surveillance. Cancer. 2012;118(4):997-1006.

\title{
Mit Brokkoli gegen Blasenkrebs?
}

\section{Das Risiko, an Blasenkrebs zu erkranken, lässt sich vielen Studien zufolge durch den Verzehr von Gemüse aus der Familie der Kreuzblütler, also etwa Brokkoli, senken. Eine Metaanalyse sollte nun prüfen, ob da was dran ist.}

n ihrer Studie werteten Ärzte von der Universitätsklinik in Hangzhou südlich von Shanghai 78 Publikationen zu diesem Thema aus. In der Endauswertung für die Metaanalyse wurden schließlich insgesamt zehn Studien berücksichtigt, fünf Fall-Kontroll-Studien und fünf Kohortenstudien. Wie die Ärzte um Liping Xie berichteten, wurde die Hälfte der Studien in Europa - in den Niederlanden, in Finnland, Schweden, Italien und Spanien -, die andere Hälfte in den USA gemacht.

Wurden die Daten aller zehn Studien berücksichtigt, lag das relative Risiko für Blasenkrebs mit 0,80 signifikant niedriger bei jenen Teilnehmern, die in Befragungen oder Interviews angaben, Gemüse aus der Familie der Kreuzblütler zu verzehren. Vergleichsgruppe waren Teilnehmer, die solches Gemüse nur selten aßen. In einer Studie zum Beispiel lag die Verzehrmenge bei mindestens 46 Gramm pro Tag, in der Vergleichsgruppe bei weniger als 16 Gramm pro Tag. Diese Studie lief von 1986 bis 1992. In einer anderen Studie über 20 Jahre kam mehr als vier
Mal versus weniger als ein Mal pro Woche solches Gemüse auf den Tisch.

Wurden für die Berechnung nur Daten der Fall-Kontroll-Studien berücksichtigt, konnte der statistisch signifikante Zusammenhang bestätigt werden, nicht dagegen, wenn ausschließlich Daten der Kohortenstudien betrachtet wurden.

Der vor Blasenkrebs schützende Effekt durch Verzehr etwa von Kohlgemüse beruht teilweise auf dem hohen Gehalt an Glucosinolaten. Das sind aus Aminosäuren bestehende schwefelhaltige Substanzen, die den charakteristischen Geschmack dieses Gemüses ausmachen. Glucosinolate sind Vorläufermoleküle der Isothiocyanate, denen eine vor Krebs schützende Wirkung zugeschrieben wird. Unter anderem halten sie bei sich schnell teilenden Zellen den Zellzyklus an und lösen Apoptose aus.

Nach Angaben der chinesischen Ärzte wurde der antiproliferative Effekt der Isothiocyanate in vitro unter anderen bei invasiven und Doxorubicin-resistenten Blasenkarzinomzellen nachgewiesen. Ers-



Gemüsesorten aus der Familie der Kreuzblütler wie Brokkoli und Blumenkohl enthalten vor Krebs schützende Substanzen, die Glucosinolate.

te Studien haben zudem ergeben, dass diese Substanzen auch die Entstehung von Blasentumoren sowie das Fortschreiten der Krebserkrankung im Tierversuch unterdrücken.

Fazit: In den vergangenen drei Dekaden haben viele epidemiologische Studien Hinweise dafür erbracht, dass der regelmäßige Verzehr von Kreuzblütlern, also etwa von Brokkoli, Blumenkohl und Rosenkohl das Risiko für Blasenkrebs senkt. Eine aktuelle Metaanalyse von Studien in den USA und Europa bestätigt jetzt diese Vermutung.

Peter Leiner

Liu B et al. The association of cruciferous vegetables intake and risk of bladder cancer: a meta-analysis. World J Urol. 2012 Mar 6. [Epub ahead of print]. 